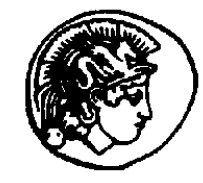

PERGAMON

International Journal of Impact Engineering 26 (2001) 497-508

www.elsevier.com/locate/ijimpeng

\title{
AFFECT OF SHOCK-INDUCED PHASE TRANSFORMATIONS ON DYNAMIC STRENGTH OF TITANIUM ALLOYS
}

\author{
YURI I. MESCHERYAKOV and ALEXANDRE K. DIVAKOV \\ Research Institute of the Mechanical Engineering Problems \\ Russian Academy of Sciences, V.O. Bol'shoi 61, Saint-Petersburg, 199178, Russia
}

\begin{abstract}
A series of complex alloyed $(\alpha+\beta)$ VT-16 Titanium alloy targets were subjected to shock loading under uniaxial strain conditions within impact velocity range of $276-600 \mathrm{~m} / \mathrm{s}$. The tests reveal a presence of forward $(\alpha \rightarrow \omega)$ and $(\beta \rightarrow \omega)$ phase transitions at the load front and reverse $(\omega \rightarrow \beta)$ transition at the release front of compressive pulse. Duration of $(\beta \rightarrow \omega)$ and $(\omega \rightarrow \beta)$ transitions is approximately $0.5 \mu$. When spallation happens after reverse ( $\omega$ $\rightarrow \beta$ ) phase transition, the spall-strength of alloy increases by $25 \%$. Oscillating regime of that transformation proves to widen the impact velocity range where the spall-strength is maximum.
\end{abstract}

Keywords: shock wave, free surface velocity, Titanium alloy, phase transformation threshold, spallation, pull-back velocity, oscillations. @ 2001 Elsevier Science Ltd. All rights reserved.

\section{NOMENCLATURE}

$\rho, \quad g / \mathrm{cm}^{3}$ - density of material,

$h_{t}, \quad m m$ - thickness of target,

$h_{i}, \quad m m$ - thickness of impactor,

$U_{\text {imp }}, m / s$ - impact velocity,

$W_{s p}, m / s$ - pull-back velocity,

$\sigma_{s p}, \quad G p a$ - spall-strength,

$W_{f}^{i}, \quad m / s \quad$ - free surface velocity threshold for the forward $(\alpha \rightarrow \omega)$ phase transformation,

$\sigma_{f}^{l}, G P a$ - stress threshold for the forward $(\alpha \rightarrow \omega)$ phase transformation,

$W_{f}^{2}, \mathrm{~m} / \mathrm{s}$ - free surface velocity threshold for the forward $(\beta \rightarrow \omega)$ phase transformation,

$\sigma_{f}^{2}, \quad G p a$ - stress threshold for the forward $(\beta \rightarrow \omega)$ phase transformation,

$W_{r}, \quad m / s$ - free surface velocity threshold for the reverse $(\omega \rightarrow \beta)$ phase transformation,

$\sigma_{r}, \quad G P a$ - stress threshold for the reverse $(\omega \rightarrow \beta)$ phase transformation,

$\mathrm{C}_{0}, \quad m / s$ - longitudinal sound speed,

$\mathrm{C}_{1}, m / s$ - plastic front velocity,

$\Delta \mathrm{t}_{\mathrm{j}}, \quad \mu s \quad$ - delay of "Plastic Wave 1", "Plastic Wave 2" or "Plastic Wave 3" relatively precursor $(\mathrm{j}=1,2,3)$,

$\Delta \mathrm{t}_{\mathrm{r}}, \mu s$ - delay of release shock of compressive pulse relatively the position of elastic precursor. 


\section{INTRODUCTION}

It has previously been shown that spall-strength of Titanium alloys is strongly dependent on the shock-induced phase transformations [1-3]. In particular, the reverse stage of phase transformation, presumably identified as the $(\omega \rightarrow \alpha)$ phase transformation, is thought to be responsible for initiating a rotational kinematical mechanism of deformation and fracture at spall zone of shock loaded Titanium alloy targets. In the present paper, we continue the investigation of physical mechanisms spallation in the Titanium alloys. It is shown that in distinction from the pure Titanium in the complex alloyed $(\alpha+\beta)$ Titanium alloys, besides the well known $(\alpha \rightarrow \omega)$ phase transformation, shock loading can induce an additional phase transition which related to the presence of $\beta$ - phase. This phase transformation we conditionally call $(\beta \rightarrow \omega)$ transition.

In this situation, the shock wave shape in the material exposed to shock-induced phase transformations, which has previously been considered by. Duvall and Graham [4], should be modified by introducing an additional forward $(\beta \rightarrow \omega)$ phase transition at the load front and reverse $(\omega \rightarrow \beta)$ transition at the release front of the compressive pulse. An example of the complicated pressure-temporal profile for the VT-16 Ti alloy is provided in Fig. 1.

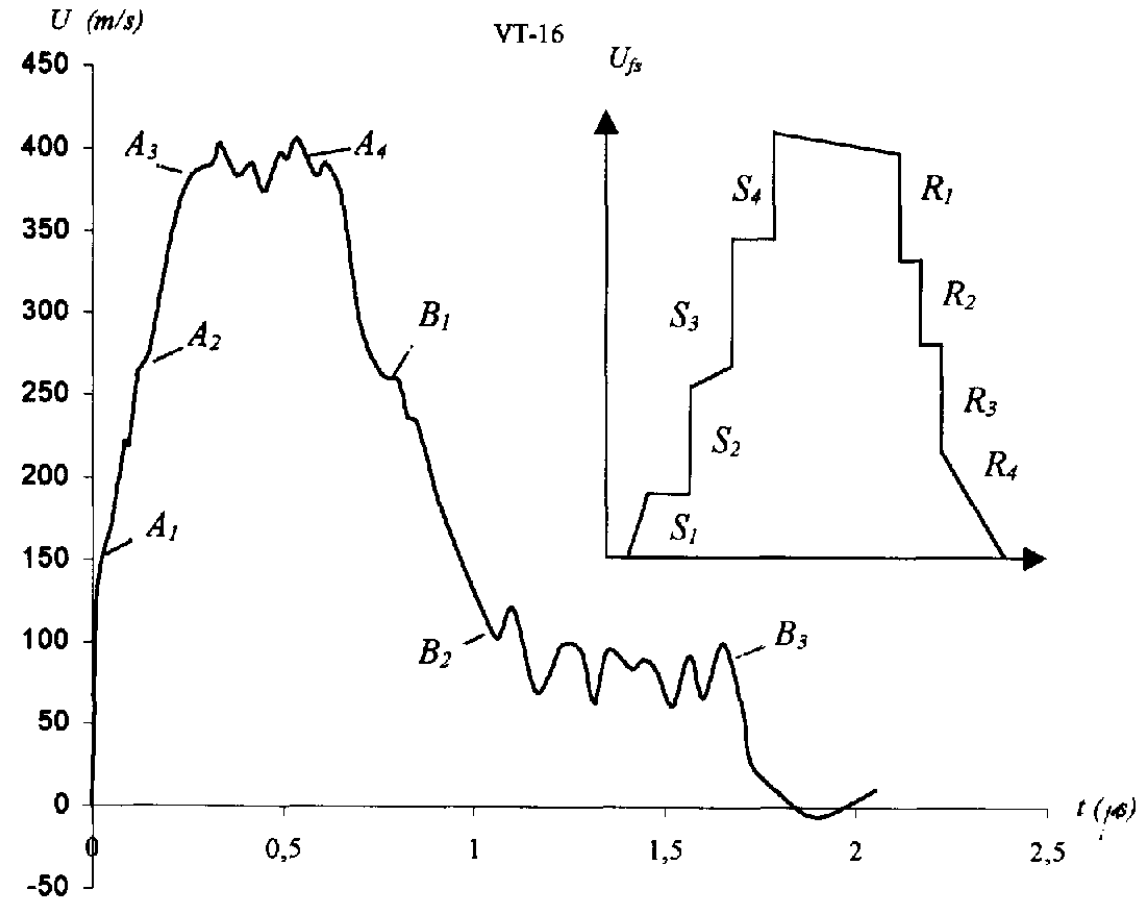

Fig. 1. Free surface velocity profile displaying forward and reverse phase transitions in VT-16 Titanium alloy under impact velocity of $409 \mathrm{~m} / \mathrm{s}$ 
Here $S_{I}$ is the elastic precursor which propagates with longitudinal sound velocity ahead of the rest of the compressive pulse. In the case of an ideal elastic-plastic solid, $S_{I}$ brings the material to the point of permanent deformation, and no deformation occurs until $S_{2}$ arrives. The second shock $S_{\boldsymbol{Z}}$ is a wave of plastic deformation called the "Plastic 1 Wave". This wave is limited in amplitude by the pressure at which the forward $(\alpha \rightarrow \omega)$ phase transition takes place. Transformation occurs in the shock front $\boldsymbol{S}_{3}$ called the "Plastic 2 Wave". This is a dilatational phase transition where the alloy density increases. In the case of pure Titanium, this increase is very small, on the order of $1.2 \%$. The second forward transition identified herein as $(\beta \rightarrow \omega)$ phase transition has a shear nature. It happens just before overstep of shock front into plateau of compressive pulse. The regions bounded by $S_{1}, S_{2}, S_{3}$ and $S_{4}$ are regions of relaxation towards equilibrium. Immediately following $\boldsymbol{S}_{\boldsymbol{1}}$ is a rarefaction fan $\boldsymbol{R}_{\boldsymbol{1}} \cdot \boldsymbol{R}_{\mathbf{2}}$ is a rarefaction shock, associated with the phase transformation which separated $S_{3}$ and $S_{4}$. Another uniform region follows $\boldsymbol{R}_{\mathbf{2}}$ and is bounded by the final rarefaction fan $\boldsymbol{R}_{\mathbf{3}}$.

Among a very wide spectrum of Titanium alloys (including Ti-6Al-4V, VT3-1, VT-6, VT14, VT-16, VT-20, VT-23 alloys with different chemical composition, oxygen content and thermal and mechanical treatments) it was found that VT-16 alloy has a maximum spall-strength $(\approx 5.4 \mathrm{GPa}$ ), its value being invariable within the more wide range of impact velocities compared to other kinds of alloys [1-3]. The present work is devoted to detailed investigation of that kind of alloy including:

- measurements of spall-strength for two regimes of tests: (i) under steady shock waves loading of plane targets of identical thickness within impact velocity range of 270 to $600 \mathrm{~m} / \mathrm{s}$, (ii) under unsteady shock waves - loading of plane targets of different thickness at the upper range of impact velocity 500 to $600 \mathrm{~m} / \mathrm{s}$.

- measurements of threshold values for phase transformation depending on the strain rate and thickness of target.

\section{CHARACTERISTIC OF MATERIALS}

VT-16 is a high-strength thermal hardened Titanium alloy of martensitic class. It is mainly used for producing rods and wire, foil and ribbon, think-walled tubes and precise profiles. Details from VT-16 are commonly manufactured by using quenching and aging. Sometimes that alloy may be used in an annealed state - for honeycomb constructions and soft tubes. In a quenched and annealed state the alloy has a high plasticity and after aging it acquires a high strength. Due to high content of Mo and $\mathrm{V}$ that alloy is effectively hardened under quenching and aging. Concrete specimens under investigation are manufactured from the forged slab, having an average grain size of $244 \mu \mathrm{m}$. Chemical composition and quasistatic strength characteristics are provided in Tables 1 and 2.

Table 1. Chemical composition of VT-16 Titanium alloy.

$\begin{array}{lllllllllllllll}\text { Element } & \mathrm{N}_{2} & \mathrm{C} & \mathrm{H}_{2} & \mathrm{Fe} & \mathrm{O}_{2} & \mathrm{Zr} & \mathrm{V} & \mathrm{Cr} & \mathrm{Mo} & \mathrm{Si} & \mathrm{Al} & \mathrm{Ti}\end{array}$

$\begin{array}{lllllllllllllll}\% & 0.05 & 0.01 & 0.002 & 0.06 & 0.15 & - & 4.5 & - & 4.8 & 0.06 & 2.9 & \text { base }\end{array}$


Table 2. Quasistatic strength-characteristics of VT-16 Titanium alloy.

\begin{tabular}{cccc}
\hline $\begin{array}{c}\text { Proportionality limit, } \\
\text { (Mpa) }\end{array}$ & $\begin{array}{c}\text { Quasistatic strength, } \\
(M p a)\end{array}$ & $\begin{array}{c}\delta \\
(\%)\end{array}$ & $\begin{array}{l}\psi \\
(\%)\end{array}$ \\
\hline & 1096 & 12.4 & 28.7 \\
\hline 870 & 109 & 12.4 \\
\hline
\end{tabular}

\section{EXPERIMENTAL APPROACH AND RESULTS}

Dynamic loading of specimens was performed with a $37 \mathrm{~mm}$ bore-diameter one stage light gas gun. Temporal profiles of the free surface velocity were recorded with two-channel velocity interferometer.

During the first stage of shock tests of VT-16 Ti alloy, a series of time-resolved free surface velocity profiles was obtained in the impact velocity range of 276 to $567 \mathrm{~m} / \mathrm{s}$. These profiles provide an exhausting information on the elastic-plastic mechanical properties of shock loaded material to be inferred. The primary interest of the first stage of investigation was to study the steady elastic-plastic waves. Therefore a thickness of targets for the first series of tests was taken to be sufficient for the elastic precursor to acquire the steady value. For VT-16 Titanium alloy, the equilibrium value of the elastic precursor is approximately $2.35 \mathrm{GPa}$ and corresponds to target thickness of $7 \mathrm{~mm}$. Special tests show that the further increase of target thickness does not lead to a decay of the elastic precursor.

Spall-strength data were obtained on the basis of interferometric measurements of pullback velocities. We avoid the use of corrections for calculations of spall-strength which have previously been introduced on the basis of appropriate mechanical model for spallation $[5,6]$. These corrections can readily be applied if the need arises. Instead we use a direct measuring of load and release plastic front velocities $\mathrm{C}^{1}$ and $\mathrm{C}^{\mathrm{r}}$ as done in [7]. Spall-strength data are presented in the form of dependencies of pull-back velocity $W_{s p}$ and spall-stress $\sigma_{s p}$ on the impact velocity $U_{\text {imp }}$ in Table 3 and in Fig. 2 .

Table 3. Results of tests for $7 \mathrm{~mm}$ VT-16 Titanium alloy targets.

\begin{tabular}{ccccccccccc}
\hline $\begin{array}{c}h_{t} \\
(\mathrm{~mm})\end{array}$ & $\begin{array}{c}h_{i} \\
(\mathrm{~mm})\end{array}$ & $\begin{array}{c}U_{i m p} \\
(\mathrm{~m} / \mathrm{s})\end{array}$ & $\begin{array}{c}W_{s p} \\
(\mathrm{~m} / \mathrm{s})\end{array}$ & $\begin{array}{c}\sigma_{s p} \\
(G P a)\end{array}$ & $\begin{array}{c}W_{f}^{l} \\
(\mathrm{~m} / \mathrm{s})\end{array}$ & $\begin{array}{c}\sigma_{f}^{I} \\
(G P a)\end{array}$ & $\begin{array}{c}W_{f}^{2} \\
(\mathrm{~m} / \mathrm{s})\end{array}$ & $\begin{array}{c}\sigma_{f}^{2} \\
(G P a)\end{array}$ & $\begin{array}{c}W_{r} \\
(\mathrm{~m} / \mathrm{s})\end{array}$ & $\begin{array}{c}\sigma_{r} \\
(G P a)\end{array}$ \\
\hline 7.05 & 2.05 & 276 & - & & 214 & 2.51 & 230 & 2.38 & 160.2 & 1.87 \\
7.05 & 2.07 & 362 & - & & 294 & 3.45 & 318 & 3.28 & 233.2 & 2.74 \\
7.05 & 2.06 & 389 & - & & 246.5 & 3.53 & 339 & 3.5 & 300 & 2.88 \\
7.05 & 2.06 & 409 & - & & 281.5 & 4.11 & 382 & 3.95 & 350 & 3.3 \\
7.07 & 2.00 & 432 & - & & 264 & 4.35 & 390 & 4.02 & 370 & 3.1 \\
7.05 & 2.04 & 452 & - & & 305.8 & 4.82 & 433 & 4.47 & 410 & 3.6 \\
7.00 & 2.06 & 508 & 458.4 & 5.38 & 337.6 & 5.17 & 465 & 4.8 & 440 & 3.96 \\
7.05 & 2.04 & 522 & 445 & 5.23 & 329.9 & 5.17 & 465 & 4.8 & 440 & 3.86 \\
7.05 & 1.99 & 531 & 448 & 5.26 & 325 & 5.05 & 463 & 4.78 & 430 & 3.82 \\
7.05 & 2.06 & 556.7 & 460.7 & 5.41 & 342.8 & 4.45 & 530 & 5.5 & 475 & 4.02 \\
\hline \hline
\end{tabular}




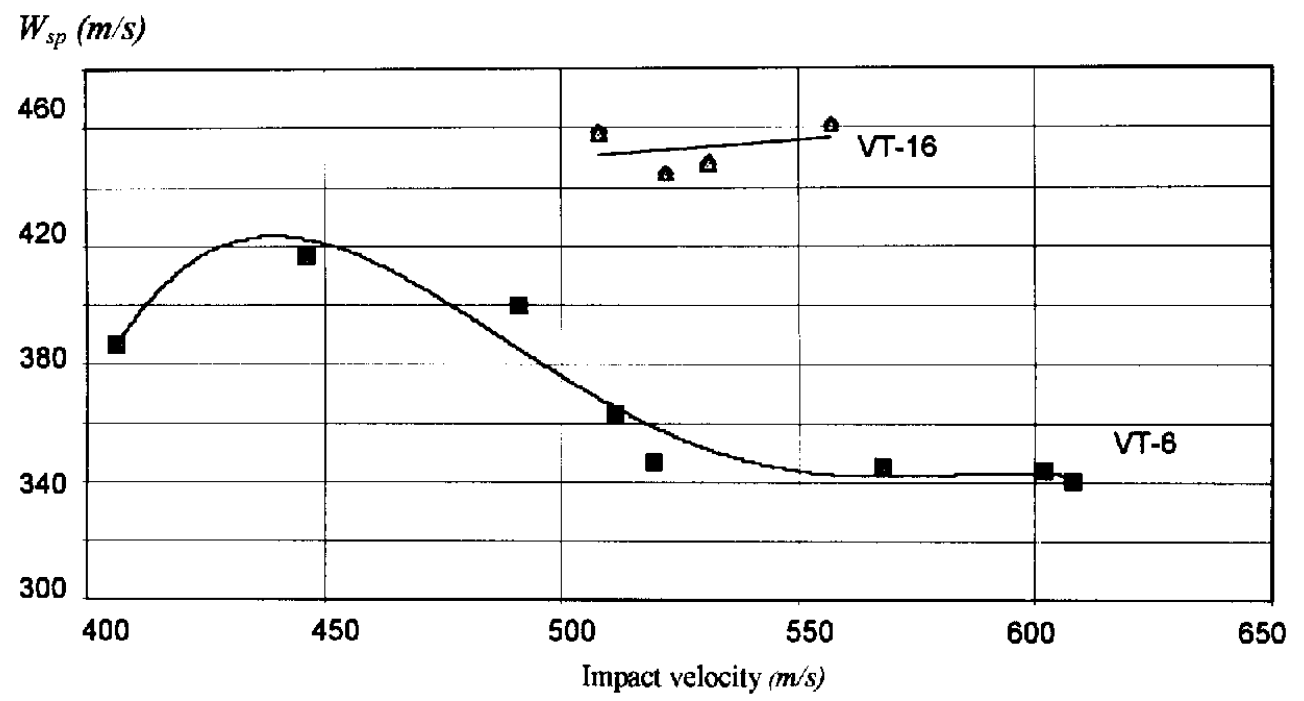

Fig. 2. Dependencies of pull-back velocity on the impact velocity for the VT-16 and VT-6 Titanium alloys .

In Fig 2 for comparison we present the pull-back velocity dependencies for two kinds of Titanium alloys - VT-16 and VT-6. Whereas the pull-back velocity for the VT-16 alloy is practically invariable within the overall impact velocity range, for the VT- 6 alloy that dependence has two distinct regions where its value differs by 80 to $100 \mathrm{~m} / \mathrm{s}$, i.e. approximately $25 \%$. Analogous behavior of spall-strength is proper for the other $(\alpha+\beta)$ Titanium alloys [1-3].

\section{SHOCK-INDUCED PHASE TRANSFORMATIONS}

The most important features of the Titanium alloy under investigation are the shock-induced phase transformations at the load and release fronts of the compressive pulse. For the pure Titanium, the load phase transformation has previously been identified as the $(\alpha \rightarrow \infty)$ transformation $[8,9]$. In the pure Titanium, the load $(\alpha \rightarrow \omega)$ phase transition is often seen as a small inclination of plastic front (point $A_{2}$ in Fig. 1) because the density of the $\omega$-phase differs from that of the $\alpha$-phase only by $1.2 \%$.

Study of shock-induced $(\alpha \rightarrow \omega)$ phase transformation in pure Titanium in [9] by using an explosive loading of specimens in special capsules could not provide conditions of all-round tension for the release stage of dynamic loading. Under these conditions approximately $70 \%$ of $\omega$-phase nucleated during the forward $(\alpha-\omega)$ phase transformation was found to be conserved in the post-shocked specimen.

Specifics of shock loading used in $[10,11]$ (three-angle compression pulse having a very steep back front) doesn't allow the second forward phase transition in $(\alpha+\beta)$ Titanium alloys to be seen at the first (load) front of the compressive pulse before its overstep into plateau (piece $A_{3}-A_{4}$ in Fig. 1). It may be identified as $(\beta \rightarrow \omega)$ phase transition related to the presence of $\beta$-phase in the initial Titanium alloy. This phase transformation requires a finite time, so its duration is equaled to $400-500 \mathrm{~ns}$. In this connection one should pay attention to the configuration of the free surface velocity profile in Fig.1. The character of signals and their duration are practically identical for pieces $A_{3}-A_{4}$ and $B_{2}-B_{3}$ so we can assume that piece $B_{2}$ $-B_{3}$ of the compressive pulse just corresponds to the reverse $(\omega \rightarrow \beta)$ phase transformation. In our study, a reverse $(\omega \rightarrow \beta)$ phase transition is confidently identified as an extensive region 
(piece $B_{2}-B_{3}$ in Fig. 1) before the rarefaction fan $R_{3}$. if a spallation happens after that transformation.

To date we cannot precisely identify the kind of shock-induced phase transformations at the load and reverse shock fronts of the compressive pulse. A large volume of serious microstructure and X-ray investigations in the post shocked specimens are needed. Therefore in this paper we conditionally call these transitions as $\beta \rightarrow \omega$ or the second forward phase transition and reverse $\omega \rightarrow \beta$ phase transition.

In our experiments we measure the threshold free surface velocities $W_{l}{ }^{1}$ and $W_{l}{ }^{2}$ for the first and second forward phase transitions in the points $A_{2}$ and $A_{3}$ and for the reverse phase transition $W_{r}$ (point $B_{2}$ in Fig. 1). These data are provided in Table 3. "Plastic wave 1", "Plastic Wave 2" and "Plastic Wave 3" velocities (denoted as $j=1,2,3$ ) which are needed for calculating the threshold stresses were determined as follows:

$$
C_{1}=h_{1} /\left(h_{t} / C_{0}+\Delta t_{j}\right)
$$

Here $h_{1}$ is the target thickness, $C_{0}$ is the longitudinal sound speed, $C_{1}$ is the plastic load front velocity and $\Delta \mathrm{t}_{\mathrm{j}}$ is the delay of "Plastic Wave 1" "Plastic Wave 2" or "Plastic Wave 3" relatively elastic precursor (see Fig 1). A value of plastic wave velocity for release shock in the $\omega$-phase $C_{r}$ has been calculated by using the following formula:

$$
C_{r}=h_{t} /\left(h_{t} / C_{l}{ }^{t}+\Delta t_{r}-2 h_{i} / C_{l}^{i}\right)
$$

where $\mathrm{h}_{\mathrm{t}}$ is the thickness of target, $\mathrm{h}_{\text {; }}$ is the thickness of impactor, $\mathrm{C}_{0}{ }^{\mathrm{t}}$ and $\mathrm{C}_{0}{ }^{\mathrm{i}}$ are the longitudinal sound velocities in the material of target and impactor, respectively. $\Delta \mathrm{t}_{\mathrm{r}}-$ is the delay of release shock of compressive pulse relatively the position of elastic precursor. Then the stress-threshold for reverse phase transition is calculated as follows:

$$
\sigma_{\mathrm{r}}=0,5 \rho \mathrm{C}_{\mathrm{r}} \boldsymbol{W}_{r}
$$

Dependencies of threshold free surface velocities for both forward and reverse phase transitions on the impact velocity are plotted together in Fig. 3 .

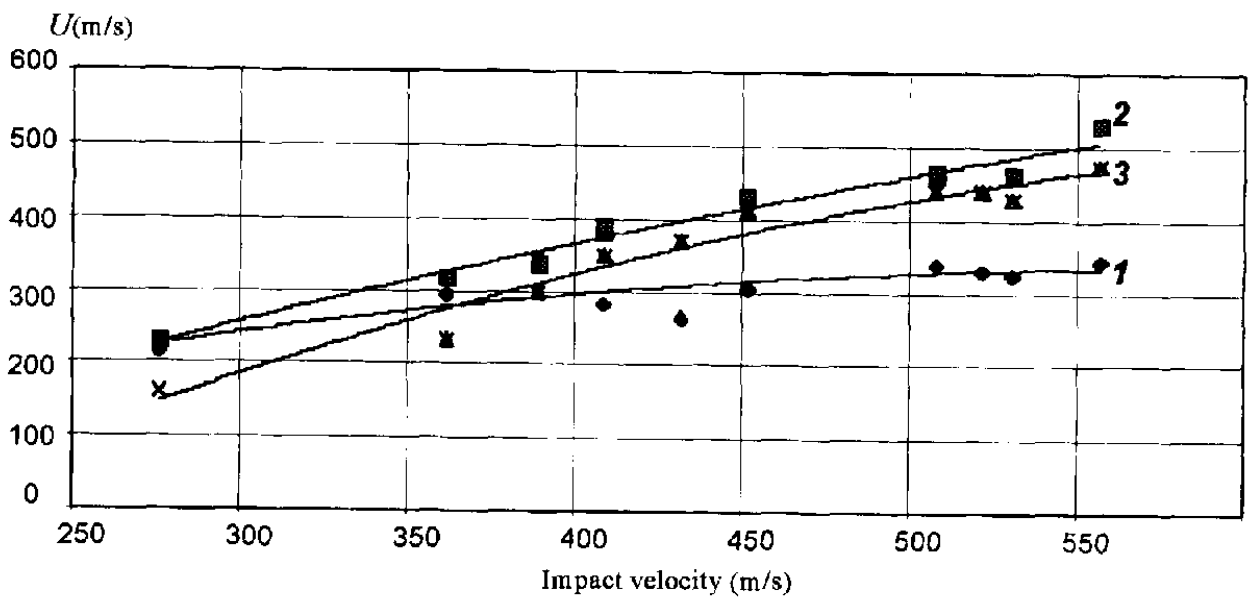

Fig. 3. Free surface velocity thresholds for the first $W_{f}^{i}(1)$ and second $W_{f}^{2}(2)$ forward and reverse $W_{r}^{2}(3)$ phase transitions as functions of impact velocity 
Conclusions following from these dependencies are:

i). Threshold for the first forward $(\alpha \rightarrow \omega)$ phase transformation is practically invariable with increasing strain rate. It slightly increases at the lower range of impact velocities but remains constant after an impact velocity of $500 \mathrm{~m} / \mathrm{s}$.

(ii) Thresholds for the second forward $(\beta \rightarrow \omega)$ and reverse $(\omega \rightarrow \beta)$ phase transformations linearly grow with the increase of impact velocity.

(iii) There is a hysteresis in threshold values for the second forward $(\beta \rightarrow \omega)$ and reverse $(\omega \rightarrow \beta)$ phase transitions.

In the second stage of tests a series of plane targets of variable thickness were shocked at the upper range of impact velocities $(500$ to $600 \mathrm{~m} / \mathrm{s}$ ). Test results are provided in Table 4.

Table 4. Results of shock tests for the second set of target

\begin{tabular}{ccccccc}
\hline $\begin{array}{c}h_{t} \\
(\mathrm{~mm})\end{array}$ & $\begin{array}{c}h_{i} \\
(\mathrm{~mm})\end{array}$ & $\begin{array}{c}U_{i} \\
(\mathrm{~m} / \mathrm{s})\end{array}$ & $\begin{array}{c}W_{s p} \\
(\mathrm{~m} / \mathrm{s})\end{array}$ & $\begin{array}{c}W_{f}^{1} \\
(\mathrm{~m} / \mathrm{s})\end{array}$ & $\begin{array}{c}W_{f}^{2} \\
(\mathrm{~m} / \mathrm{s})\end{array}$ & $\begin{array}{c}W_{r} \\
(\mathrm{~m} / \mathrm{s})\end{array}$ \\
\hline 2.30 & 1.50 & 533.8 & 342.9 & 440 & - & - \\
3.23 & 1.50 & - & 391.8 & 475 & 525 & 386 \\
4.17 & 1.52 & 542.8 & 381.9 & 420 & 475 & 350 \\
4.18 & 1.50 & 566.6 & 368.2 & 462 & 480 & 352.5 \\
4.24 & 1.50 & 506.8 & 394 & 425 & 452 & 330 \\
5,19 & 1.81 & 593 & 370 & 434.5 & 520 & 357.3 \\
5.24 & 1.80 & 583 & 357.3 & 454 & 525 & 362.3 \\
6.35 & 1.94 & 530 & 425 & 370 & 490 & 335 \\
\hline
\end{tabular}

In Fig. 4 we present the dependencies of pull-back velocity and phase transition thresholds on the impact velocity for $4 \mathrm{~mm}$ VT-16 targets.

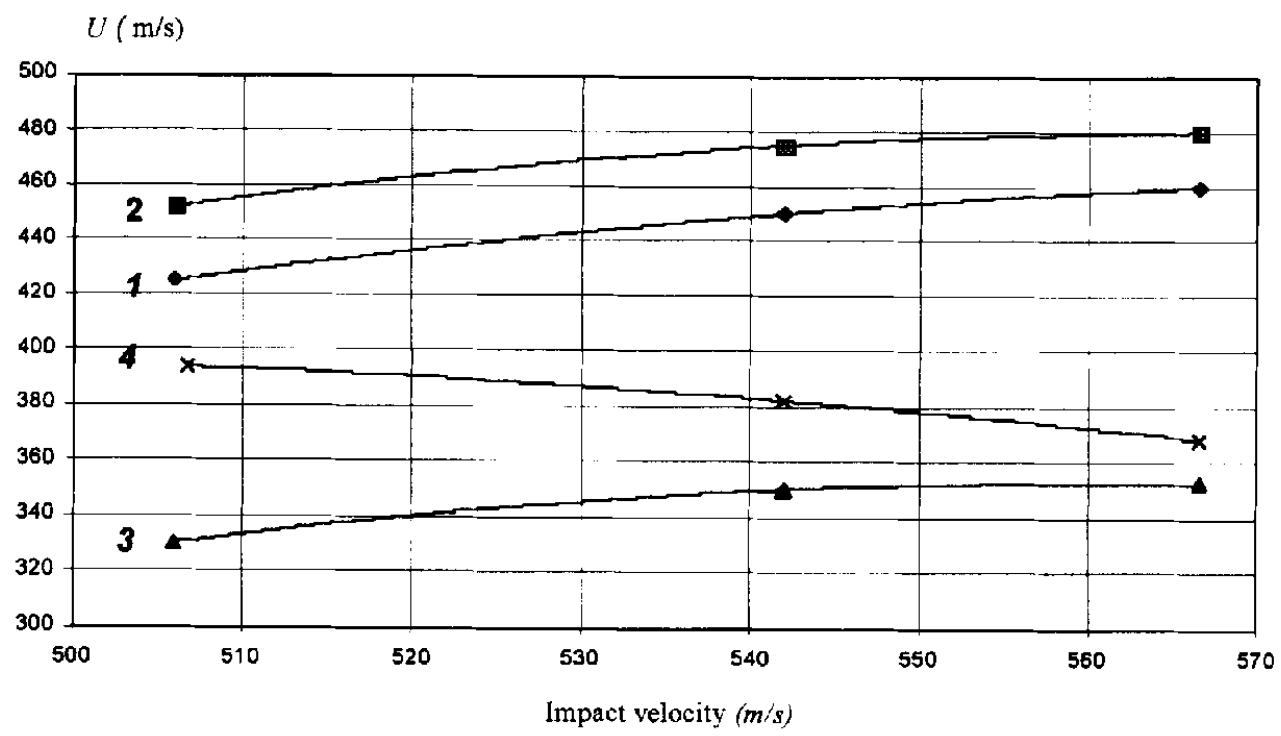

Fig. 4. Thresholds for the first $W_{f}^{\prime}(I)$ and second $W_{f}^{2}(2)$ forward and reverse $W_{r}^{2}$ (3)phase transitions and pul-back velocity $W_{s p}(4)$ for the second set of VT-16 alloy targets. 
One can see that spall-strength of VT-16 alloy drops with the increase of the impact velocity (compare the invariable value of spall-strength for $7 \mathrm{~mm}$ targets in Fig.2). In this figure we also present the forward and reverse phase transition thresholds as a functions of impact velocity. These dependencies show a growth of the threshold values not only for the second forward $(\beta \rightarrow \omega)$ and reverse $(\omega \rightarrow \beta)$ phase transitions, but also for the first forward $(\alpha \rightarrow \omega)$ phase transformation. In the case of $7 \mathrm{~mm}$ targets the latter dependence was almost invariable (and accordingly, the spall-strength also does not change within the impact velocities under investigation).

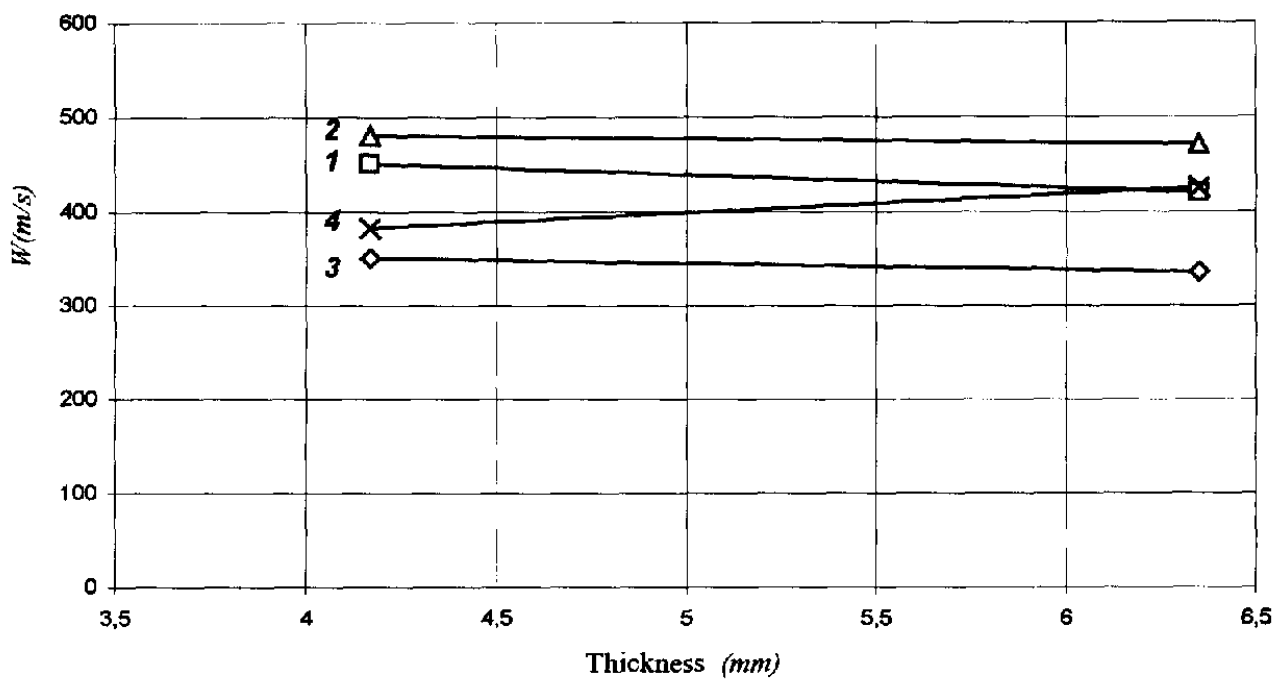

Fig. 5. Thresholds for the first $W_{l}^{l}(1)$, second $W_{l}^{2}(2)$ forward, reverse $W_{r}(3)$ phase transitions and pull-back velocity $W_{s p}(4)$ versus target thickness

In Fig. 5 we present dependencies of the phase transition thresholds and pull-back velocity on the target thickness. The phase transition thresholds decrease with the increase of the target thickness whereas the pull-back velocity increases. For the $6.35 \mathrm{~mm}$ target the pull-back velocity approaches its value for $7 \mathrm{~mm}$ target.

\section{DISCUSSION}

Comparison of the time-resolved profiles for four target in Fig. $6(a-d)$ shows a very distinct coupling between reverse phase transformation and spall-strength. It is clearly seen that spallstrength appreciably increases if the reverse phase transformation occurs before the spallation. The same has been found for other kinds of Titanium alloys [1-3]. In these alloys dependence of spall-strength can be subdivided by two regions. The first region corresponds to impact velocities lower 500 to $520 \mathrm{~m} / \mathrm{s}$. In this region the reverse phase transformation happens before the spallation. Accordingly, the material reveals the greatest spall-strength. With the increase of impact velocity the time-delay for spallation decreases whereas the time-delay for the reverse phase transition remains to be invariable. After impact velocities on the order of $500-520 \mathrm{~m} / \mathrm{s}$ thespallation happens before the reverse phase transition. In this case, pull-back velocity noticeably decreases. The nature of that phenomenon is still poorly understood. The affect of reverse phase transformations on dynamic strength of Titanium alloys proves to be indirect. After 


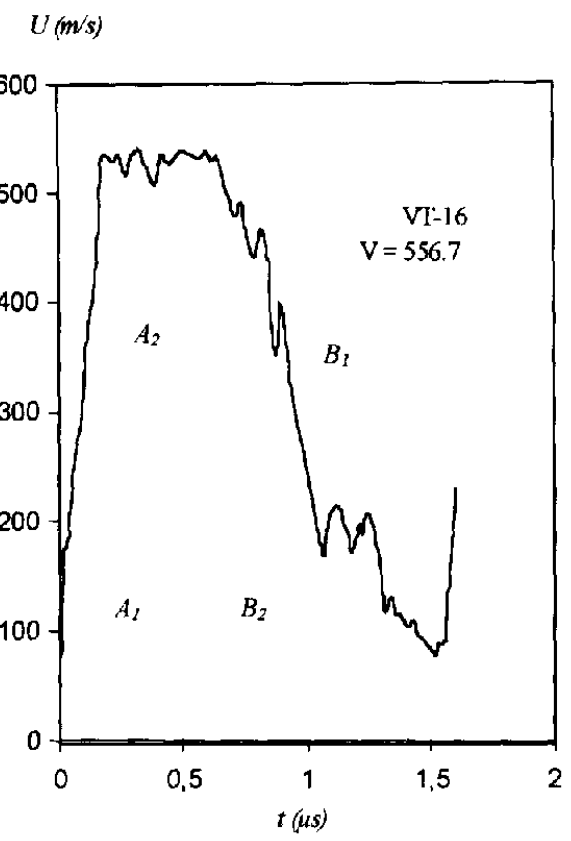

a)

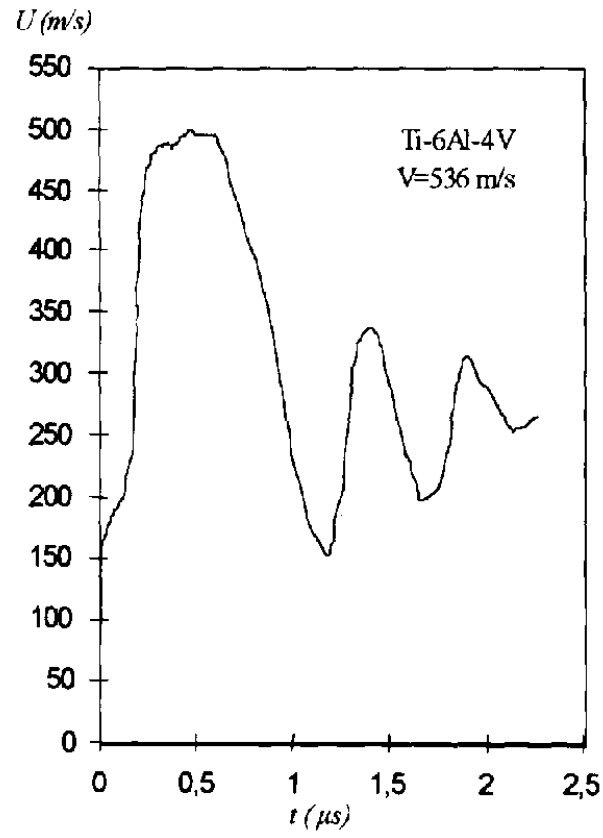

c)
$U\left(m^{\prime} s\right)$

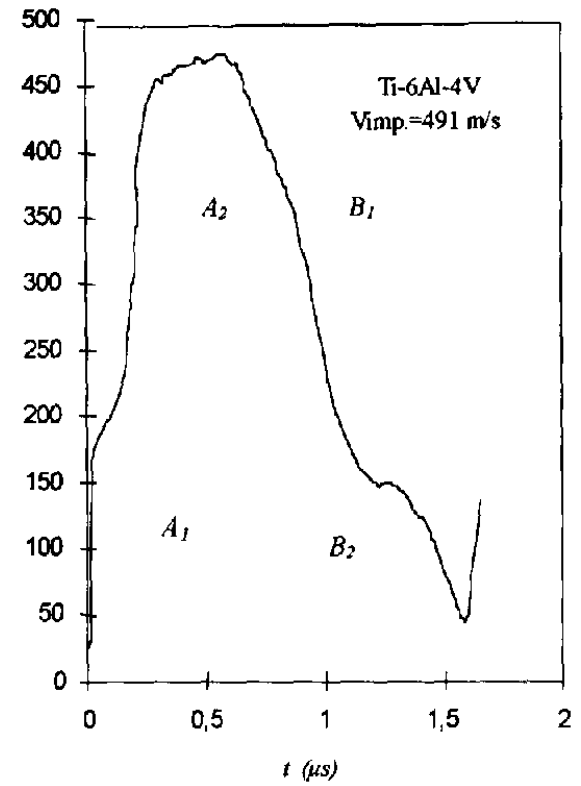

b)

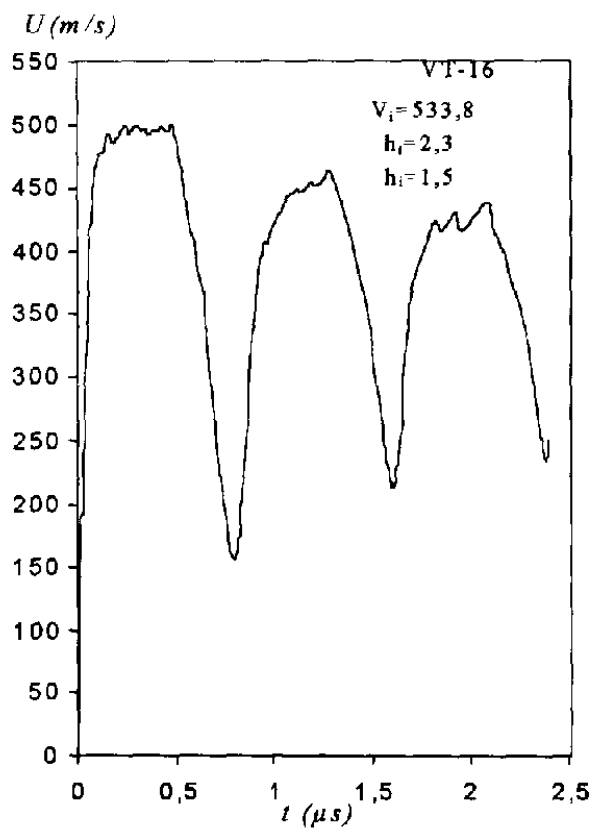

d)

Fig.6. (a) - free surface velocity profile in VT-16 alloy for the case when phase transition happens before spallation. (b) - the same for Ti-6Al-4V alloy. (c) profile for the case when spallation happens before phase transition, (d) - profile for the case of artificial spallation 
microstructure investigations of post-shocked specimens the numerous rotational formations of mesoscopic scale level were found within spall zone [1-3]. The rotational kinematic mechanism at the mesolevel is thought to be responsible for the more effective dissipation of the shock wave energy at the spall zone of targets. This mechanism is believed to be realized only during the phase transformation process because of lowering the threshold for the rotation nucleation owing to unstable state of material.

The affect of reverse phase transformation on the spall-strength of Titanium alloys depends on both the intensity of the reverse phase transformation process and its duration, both these characteristics being dependent on strain rate and kind of alloy. This can be seen by comparing results of spall tests under identical conditions of two kinds of Ti alloys targets, Ti- $6 \mathrm{~A}-4 \mathrm{~V}$ and VT-16, presented in Fig. $6 a, b$. The character of the first forward $(\alpha \rightarrow \omega)$ phase transition for both kinds of alloys is identical. At the free surface velocity profiles that transition looks as small change of the plastic front slope (point $\left.A_{2}\right)$. As for the second forward $(\beta \rightarrow \omega)$ phase transition its character for VT-16 and Ti-6Al-4V alloys differs. In the VT-16 Ti alloy that transition has an oscillating nature whereas in the Ti-6Al-4V alloy it is sufficiently smooth and inert. Besides, in the VT-16 alloy that transition flows longer than in the Ti-6Al-4V. Comparison of a series of the free surface velocity profiles for two alloys shows that in the VT-16 the reverse trarsition is realized in the wide range of impact velocities from $276 \mathrm{~m} / \mathrm{s}$ through $593 \mathrm{~m} / \mathrm{s}$, while in the Ti-6Al$4 \mathrm{~V}$ alloy it disappears after impact velocity of $510 \mathrm{~m} / \mathrm{s}$.

In reality both features, duration of the reverse phase transition and its realization in the more wide range of strain rates are the functions of the intensity of $(\beta \rightarrow \omega)$ phase transformation. Phase transformation is generally known to be a very effective relaxation process. Under certain intensity of the phase transformation, the relaxation process may acquire an oscillating character. This happens if the transformation-induced unloading is faster than growth the external (applied) stress. In this case material unloads so quickly that the reason which causes the phase transformation disappears. After that internal stresses increase again resulting in a new stage of phase transformation. That process repeats itself periodically and continues until the initial phase expends. Thus, high intensity of the phase transformations in VT-16 $\mathrm{Ti}$ alloy induces the oscillating regime of phase transformation, which, in turn, results in increasing the duration of phase transformation process and thereby in creating the favorable conditions for the nucleation of rotational structures.

In distinct from the $(\alpha \rightarrow \omega)$ transformation, the $(\beta \rightarrow \omega)$ phase transformation is mainly realized by means of shear. Nevertheless all-round conditions of tension created within the spall zone during spallation also help its realization. To check this we performed one additional experiment with the so-called artificial spallation, where interference of rarefaction waves happens outside the target body. The free surface profile for that test is provided in Fig. $6 d$. One can see an absence of reverse phase transition at the release front of the compressive pulse. This means that both ( $\omega$ $\rightarrow \alpha)$ and $(\omega \rightarrow \beta)$ reverse phase transformations can be realized only under condition of allround tension.

Pay attention once more interesting phenomenon related to the forward and reverse $(\beta \leftrightarrow \omega)$ phase transformations. Formal deciphering of interference signal often gives a resulting free surface profile for which the load front turns out to be shorter then release front of compressive pulse. Typical case is presented in Fig. 7. One can see that pick velocity smaller than impact velocity under symmetrical collision by $30 \mathrm{~m} / \mathrm{s}$ At the same time release front longer approximately by the same value. This means that kinetic energy of external loading, which has been spent on the $(\beta \rightarrow \omega)$ phase transformation at the load front, returns back during the $(\omega \rightarrow \beta)$ reverse phase transformation at the release front of compressive pulse. To provide that returning a spallation must be realized after the second reverse phase transformation. 


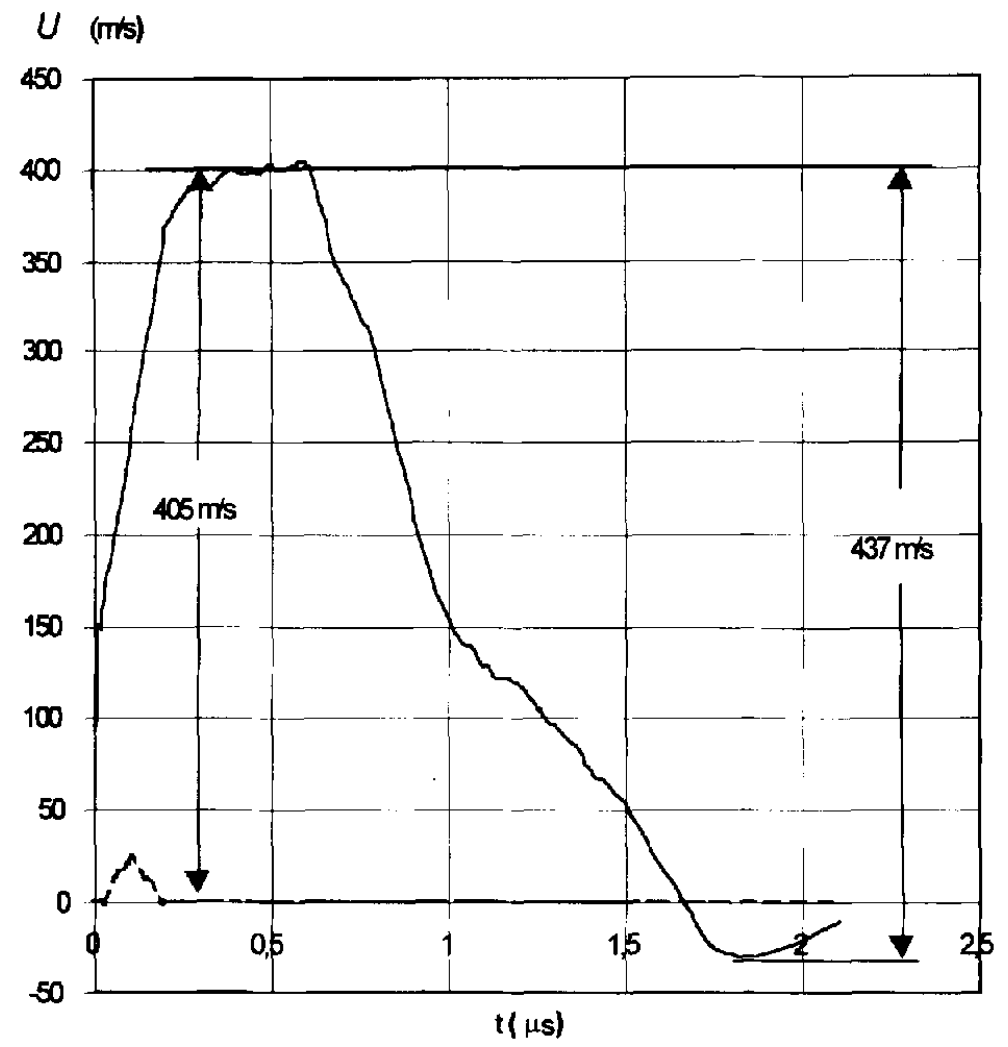

Fig.7. Free surface velocity profile in VT-16 $\mathrm{Ti}$ alloy displaying a decrease of velocity at the load front and increase of velocity at the release front $\left(U_{i m p}=437 \mathrm{~m} / \mathrm{s}\right)$.

The analysis presented above shows that spall-strength of Titanium alloys is sensitively depend on the intensity of both forward and reverse phase transformations flowing during the shock wave propagation in the shock compressed material. In this situation it seems to be profitable to use the kind of alloy which could provide the greatest intensity of the phase transformation processes.

\section{CONCLUSIONS}

Experimental investigations of VT-16 and some other Titanium alloys show:

- Beside the well-known shock-induced $(\alpha \rightarrow \omega)$ phase transformation which was found to be proper for the pure Titanium, complex alloyed $(\alpha+\beta)$ Titanium alloys reveal an additional shock-induced phase transformation related to presence of $\beta$ - phase, which conditionally identified as $(\beta \rightarrow-\omega)$ transformation.

- Shock-induced $(\beta \rightarrow \omega)$ phase transformation has two regimes of its realization:(i) smooth and (ii) oscillating. The latter regime results from the high-intensity stress relaxation caused by that phase transformation.

- Spall-strength of Titanium alloys sensitivitely depends on the temporal position of spallation relatively the reverse $(\omega \rightarrow \beta)$ phase transformation. When spallation happens after the phase 
transition spall-strength proves to be by $25 \%$ higher than in the opposite case, i.e. when spallation occurs before the reverse phase transition.

- Oscillating regime of phase transformation appears to widen the strain-rate range where dynamic strength of Titanium alloys is maximum.

Acknowledgment--This work was supported by Army Research Laboratory, Contract DAAL01-98-P-0796

\section{REFERENCES}

[1] Mescheryakov Yu.I., Divakov A.K., Zhigacheva N.I, Petrov Yu. A..Shock-induced $\alpha$ - $\omega$ phase transformation and mechanisms of spallation in shock loaded Titanium alloys. Proc. of APS-Conf. "Shock Compression of Condensed Matter-1999", Snowbird., Uh, 1999: p 439-442.

[2] Mescheryakov Yu.I., Divakov A.K., Zhigacheva N.I Shock-induced phase transformation and vortex instabilities in shock loaded titanium alloys. Intern. $J$. of Shock Waves. 2000; 10 (1): 43-56.

[3] Mescheryakov Yu.I., Divakov A.K., Zhigacheva N.I, Petrov Yu. A. Spall strength and phase transformation in Titanium alloys in impact loading. The Physics of Metals and Metallography. 1999; 87 (5): 443-449.

[4] Duvall] G.E, Graham R.A. Phase transitions under shock-wave loading Review of Modern Physics. 1977; 49(3): 523-579.

[5] Romanchenko V.I., Stepanov G.V J. of Appl. Mech. and Tech.Phys. 1980; 4 : 141 (Russian).

[6] Kanel G.I., Rasorenov S.V., Fortov V.E. Doklady Akademii nayk USSR. 1987; 294 (2): 350 (Russian).

[7] Barker L.M., Lundergun C.D., Herrman W. Dynamic Response of Aluminum. J. of Appl. Phys. 1964;35 (4): 1203-1212.

[8] Rasorenov S.V., Utkin A. V., Kanel, G.I., Fortov V. E., Yarunichev A.S., Baumund K., Karow H.U. High Pressure Research. 1995; 13: 367-376.

[9] A.R. Kutsar, V.N. German, G.I. Nosova, $\alpha-\omega$ phase transition in the Titanium and Zirconium in snock waves. Doklady Academii Nauk (Russia). 1976; 213b(1): 81-84.

[10] Kanel' G.I., Rasorenov S.V., Fortov V.E. J. Tech. Phys. 1986; 56(3): 586.

[11] Kanel' G.I., Rasorenov S.V., Fortov V.E. Doklady Akademii nauk USSA, 1987; 294(2): 350. 